\title{
Mucin-type sialyl-Tn antigen is associated with PD-L1 expression and predicts poor clinical prognosis in breast cancer
}

\author{
Feng $\mathrm{Xu}^{1} \wedge$, Hongying $\mathrm{Zhao}^{2},{\mathrm{Jie} \mathrm{Li}^{1} \text {, Hongchuan Jiang }}^{1}$ \\ ${ }^{1}$ Department of Breast Surgery, Beijing Chao-Yang Hospital, Beijing, China; ${ }^{2}$ Department of Pathology, Beijing Chao-Yang Hospital, Beijing, China \\ Contributions: (I) Conception and design: F Xu; (II) Administrative support: H Jiang; (III) Provision of study materials or patients: H Zhao, J Li; (IV) \\ Collection and assembly of data: F Xu, H Zhao, J Li; (V) Data analysis and interpretation: H Jiang, F Xu; (VI) Manuscript writing: All authors; (VII) \\ Final approval of manuscript: All authors. \\ Correspondence to: Feng Xu. Department of Breast Surgery, Beijing Chao-Yang Hospital, Capital Medical University, No. 8 Gongti Nan Lu, \\ Chaoyang District, Beijing 100020, China. Email: drxufeng@163.com.
}

Background A recent study showed that mucin-type sialylated O-linked glycans could induce the
increased expression of PD-L1 via binding to Siglec receptors. However, the relationship between the
expression of the mucin-type sialyl-Tn antigen (sTn) and PD-L1 remains unclear in breast cancer (BC).
Therefore, we investigate the clinicopathological and prognostic effects of sTn expression and its relationship
with PD-L1 expression in BC tissues.

Methods: We retrospectively analyzed the clinical data of 380 invasive BC patients between January 2011 and January 2014. The last follow-up time was January 31, 2019 with a median follow-up of 62 months. The expression of the sTn antigen and PD-L1 in 380 tumor specimens was assessed by immunohistochemistry. Correlations between sTn/PD-L1 expression and clinicopathological features and prognoses were analyzed.

Results: In BC tissues, the positive expression rate of PD-L1 (20.5\%) was much lower than that of sTn (41.8\%). Pearson's contingency analysis showed that sTn and PD-L1 expression in tumor tissues demonstrated a high correlation $(\mathrm{P}<0.001)$. High $\mathrm{s}$ Tn expression was associated with negative ER expression $(\mathrm{P}<0.001)$, positive HER-2 status $(\mathrm{P}<0.001)$, advanced tumor stage $(\mathrm{P}<0.001)$, high density of CD8+ tumorinfiltrating lymphocytes (TILs) $(\mathrm{P}=0.028)$, and positive lymph node metastasis $(\mathrm{P}=0.002)$. Moreover, patients with concomitant high expression of both markers had the highest risk of relapse $(\mathrm{P}<0.001)$ and mortality $(\mathrm{P}<0.001)$. The multivariate Cox regression model revealed that positive sTn expression (HRos: 1.941, 95\% CI: 1.168, 3.223, Pos=0.028; HRpfs: 1.739, 95\% CI: $1.063,2.847$, Ppfs=0.010) and positive PD-L1 expression (HRos: 1.912, 95\% CI: 1.138, 3.212, Pos=0.017; HRpfs: 1.863, 95\% CI: 1.116, 3.110, Ppfs=0.014) were independent indicators for poor overall survival (OS) and progression-free survival (PFS), respectively. Conclusions: BC patients who expressed both sTn and PD-L1 had poorer survival. Therefore, combinational therapy with dual blockade might benefit BC patients with sTn(+)/PD-L1(+) expression, which requires further examination in future clinical trials.

Keywords: STn; programmed cell death-ligand 1 (PD-L1); breast cancer (BC); prognosis; immunotherapy

Submitted Feb 07, 2021. Accepted for publication May 21, 2021.

doi: $10.21037 / g s-21-83$

View this article at: https://dx.doi.org/10.21037/gs-21-83

$\wedge$ ORCID: 0000-0002-5709-0188. 


\section{Introduction}

Breast cancer (BC) remains the most common malignant tumor and maintains the highest mortality among women worldwide (1). Despite great progress in individualized and targeted treatments in recent years, patients with advanced BC still develop metastases and die. Due to the high mutagenic ability and heterogeneity of cancer cells, resistant genes emerge in most cases, thus causing tumor progression. Therefore, novel strategies are needed to improve the survival of patients.

As the effect of the tumor immune microenvironment on therapeutic responses has been demonstrated, the role of immunity in BC development has emerged in the past decade (2). The management of non-small cell lung cancer (NSCLC) and metastatic melanoma has opened up novel approaches for tumor immunotherapy. Programmed cell death-1 (PD-1)/programmed cell death-ligand 1 (PD-L1) is the main checkpoint for the immune response pathway in tumor's microenvironment. Recently, a group of clinical studies has shown that PD-L1 is overexpressed in various malignancies, including melanoma, NSCLC, clear cell renal cell carcinoma (CCRCC), and BC, closely related to the poor prognosis of patients (3-6). At present, the expression of PD-L1 in tumor or infiltrating immune cells remains the optimal predictor of clinical response to $\mathrm{PD}$ L1 inhibition (7). While PD-1/PD-L1 targeted therapy has shown promising results, only a few patients received better outcomes from this therapy (8). In other words, it is not possible to predict the clinical response to anti-PD-1/PDL1 therapy solely by the expression of PD-L1.

Aberrant $\mathrm{O}$-glycosylation can play a crucial role in cancer development, angiogenesis and metastasis. These alterations of abnormal glycosylation in cancer cells can result in the exposure of the oncofetal sialyl-Tn (sTn) antigen (9). The sTn antigen is a truncated O-glycan containing a sialic acid-2,6 linked to GalNAc-O-Ser/ Thr. STn overexpression has been described in various kinds of epithelial cancer, including pancreatic, ovarian, colorectal, lung, cervical, esophagus, gastric, and BC $(10-17)$. It has been reported that sTn is carried by multiple glycoproteins and is related to an immunosuppressive microenvironment (18). Recently, relevant studies demonstrated that MUC1-associated sTn antigens on cancer cells bound to Siglec-9 caused the increased expression of the checkpoint ligand PD-L1 (19,20). However, as far as we know, the relationship between the expression of sTn and PD-L1 in BC is still unclear.
Hence, we assessed the expression of s'Tn and PD-L1 by immunohistochemistry in BC tissues and explored the relationship of $s$ Tn and PD-L1 expression with clinicopathological characteristics and prognosis.

We present the following article in accordance with the REMARK reporting checklist (available at https://dx.doi. org/10.21037/gs-21-83).

\section{Methods}

\section{Patients and clinicopatbological information}

Approved by the Institutional Ethical Committees of Beijing Chaoyang Hospital (project number 2020-3-16-13), we developed and implemented a retrospective clinical research study that enrolled 380 patients with BC between January 2011 and January 2014. The detailed inclusion criteria were as follows: (I) patients were diagnosed with invasive BC for the first time; (II) tumor stage of I, II or III; (III) no history of preoperative radiotherapy and chemotherapy; (IV) clinicopathological variables including tumor size, ER/PR/ HER-2 status and histological grade were comprehensive; (V) follow-up information was comprehensive; (VI) patients did not have an autoimmune disease, other malignancies, or severe hepatic and renal dysfunction. The participants gave informed consent before taking part. All available formalin-fixed, paraffin-embedded (FFPE) specimens were independently examined by 2 experienced pathologists blinded to all patient-related information. The following covariates were measured during the baseline clinical exam and survey: age, tumor size, histological grade, ER/PR/ HER-2 status, tumor stage and lymph node metastasis. The study was conducted in accordance with the Declaration of Helsinki (as revised in 2013).

\section{Immunobistochemistry (IHC)}

The expression of s'Tn, PD-L1, CD4, and CD8 in primary and metastatic BC tissues was determined through IHC. FFPE archived tissues were cut into 5- $\mathrm{mm}$ thick slices, dewaxed, rehydrated, and blocked with $3 \%$ hydrogen peroxide. The sections were heated for antigen retrieval and then incubated with anti-sTn antibody (mouse monoclonal antibody, clone B72.3, dilution: 1:100, Santa Cruz), antiPD-L1 (mouse monoclonal antibody, clone 22C3, dilution: 1:200, Dako), anti-CD4 (rabbit monoclonal antibody, clone EPR19514, dilution: 1:200, Abcam), and anti-CD8 (rabbit monoclonal antibody, clone D8A8Y, dilution: 
1:200, Cell Signaling Technology) overnight at $4{ }^{\circ} \mathrm{C}$. The sections were washed with PBS and incubated with a horseradish peroxidase-labeled secondary antibody for $30 \mathrm{~min}$. Subsequently, all sections were visualized with a DAB kit, and the nucleus was counterstained with hematoxylin. For positive controls, samples of CosmcKO MCF-7 BC cells (21) and human placenta were used for sTn and PD-L1 staining, respectively. We prepared negative controls by using PBS as a substitute for the primary antibody. The results are shown in Figure S1.

\section{Evaluation of STn, PD-L1 and tumor-infiltrating lymphocyte (TIL) subsets}

We semi-quantitatively evaluated the expression of sTn and PD-L1 according to the staining intensity and distribution. Staining intensity was scored as follows: 0 (negative), 1 (weak staining), 2 (moderate staining), and 3 (strong staining). The percentage of positive cells was rated as $0,1(<5 \%)$, $2(\geq 5 \%)$, and $3(\geq 50 \%)$. Histochemistry score $=\mathrm{I} \times \mathrm{R}$. The staining intensity of sTn was scored according to our previous study (15). The cut-off value of PD-L1 expression less than $5 \%$ stained tumor cells was classified as negative and the rest as positive expression (22). We used ImagePro Plus 6.0 software to analyze the average optical density of positive expression in each field of vision. TIL subsets in the primary $\mathrm{BC}$ lesions were digitally quantified on scanned whole slide images of CD4 and CD8 stained sections, as the number of TIL/unit area (square millimeter) (22).

\section{Assessment of prognosis and follow up}

All patients were followed up through outpatient and inpatient reexamination and telephone interviews. The follow-up information included local recurrence, distant metastasis, and death. The last follow-up time was January 31,2019 , and the median follow-up time was 62 months (10-94 months). We defined overall survival (OS) as the interval between the time of breast operation and death. Progression-free survival (PFS) was defined as the time from the date of randomization to disease progression or death, censoring for loss to follow-up.

\section{Statistical analysis}

We used SPSS software (version 23.0; IBM, Armonk, NY) to analyze our data. We used either Fisher's exact test or the $\chi^{2}$ test to detect the association between immunostaining markers and clinicopathological variables. Pearson's contingency analysis analyzed the correlation between sTn and PD-L1. Co-expression of sTn and PD-L1 referred to concomitant high expression levels of both markers. Survival rates were determined using Kaplan-Meier analysis, and the differences were compared using a doublesided log-rank test. The Univariate Cox regression model was used, taking covariates (all clinicopathological features) into account. All variables with $\mathrm{P}<0.05$ were included in the multivariate Cox model. Adjusted hazard ratios (HRs) and corresponding $95 \%$ confidence intervals (CIs) were reported for variables in the multivariate model. In all cases, a two-sided $\mathrm{P}<0.05$ was considered statistically significant.

\section{Results}

\section{Association between sTn expression and clinicopathological features}

Mucin-type sTn antigen was distributed diffusely and was mainly present in the cytoplasm and/or cellular membrane of tumor cells. PD-L1 was also mostly present in the membrane and/or cytoplasm of tumor cells (Figure 1). STn and PD-L1 showed high expression rates in BC tissues, with values of $41.8 \%(159 / 380)$ and $20.5 \%(78 / 380)$, respectively. The high sTn expression was closely related to negative ER expression $(\mathrm{P}<0.001)$, positive HER-2 status $(\mathrm{P}<0.001)$, advanced tumor stage $(\mathrm{P}<0.001)$, high density of $\mathrm{CD}^{+}$TILs $(\mathrm{P}=0.028)$ and positive lymph node metastasis $(\mathrm{P}=0.002)$ (Table 1). Nevertheless, no significant statistical relation was found between the expression of sTn and other clinicopathological features such as age, tumor size, histological grade, vessel embolus, perineural invasion, $\mathrm{CD}^{+}{ }^{+}$TILs and PR status. Pearson's contingency analysis verified the positive relevance between the expression of sTn and PD-L1 ( $\mathrm{r}=0.375, \mathrm{P}<0.001)$.

\section{Survival analysis}

Kaplan-Meier survival analysis of the $380 \mathrm{BC}$ samples (Figure 2) showed markedly poor OS $(\mathrm{P}<0.001)$ and $\mathrm{PFS}$ $(\mathrm{P}<0.001)$ for the $\mathrm{s} T \mathrm{n}$ positive-expression group in contrast with the negative expression group. Moreover, the PDL1 positive expression group showed both shorter OS $(\mathrm{P}<0.001)$ and $\mathrm{PFS}(\mathrm{P}<0.001)$ compared with the negative expression group. Considering the positive relevance between the expression of sTn and PD-L1, we attempted to explore the prognostic impact of sTn/PD-L1 co-expression 


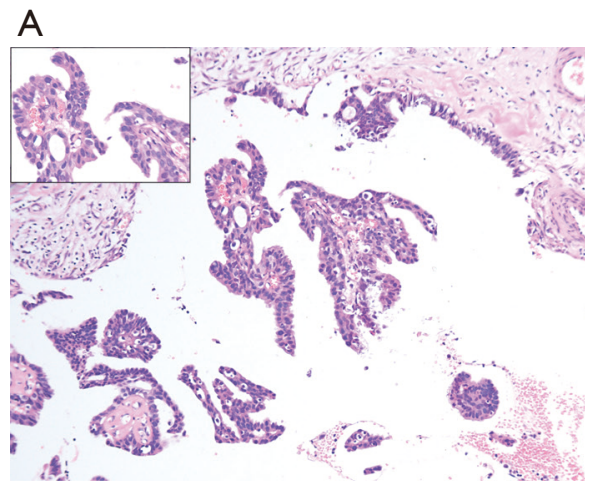

HE
B

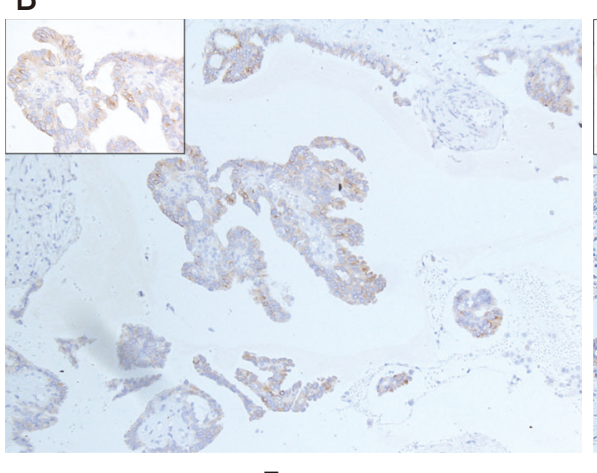

sTn

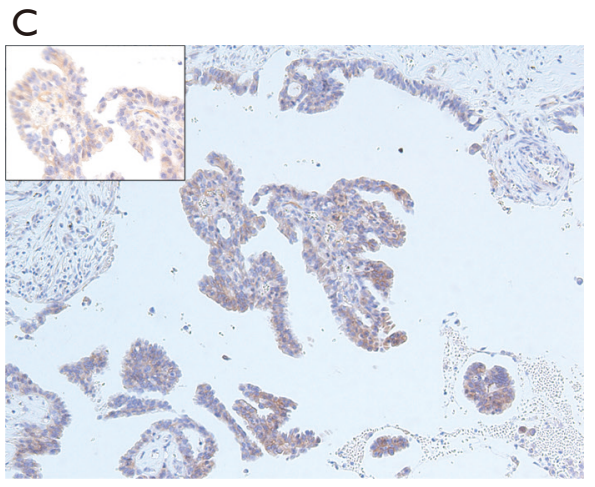

PD-L1

Figure 1 Representative examples of coexisting sTn and PD-L1 expression in primary breast cancer; (A) H\&E-stained image; (B) sTn expression. (C) PD-L1 expression. Insets show staining at $\times 40$ magnification.

in BC. Therefore, we further classified the patients into 3 groups: group A was $\sin (+) / \mathrm{PD}-\mathrm{L} 1(+)$; group B was sTn(+)/PD-L1(-) or sTn(-)/PD-L1(+); group C was $s \operatorname{Tn}(-) / \mathrm{PD}-\mathrm{L} 1(-)$. Patients in group A showed the worst OS $(\mathrm{P}<0.001)$ and PFS $(\mathrm{P}<0.001)$ among all groups (Figure 2). Given the prominent correlation between sTn and PD-L1, we completed multiple univariate and multivariate Cox proportional hazards analyses (Tables 2,3). The multivariate Cox regression model revealed that positive sTn expression (HR: 1.941, 95\% CI: 1.168, 3.223; P=0.028; HR: 1.739, 95\% CI: 1.063, 2.847; $\mathrm{P}=0.010$ ), positive PD-L1 expression (HR: 1.912, 95\% CI: 1.138, 3.212; $\mathrm{P}=0.017$; HR: 1.863 , 95\% CI: 1.116, 3.110; $\mathrm{P}=0.014$ ), and advanced tumor stage (HR: 1.805 , 95\% CI: 1.062, 3.065; $\mathrm{P}=0.029$; HR: 1.940, 95\% CI: 1.133, 3.322; $\mathrm{P}=0.016)$ were independent indicators for poor OS and PFS, respectively.

\section{Expression of STn and PD-L1 in primary and metastatic $B C$}

In total, 380 primary lesions and 68 metastatic lesions were assessed for the expression of sTn and PD-L1. STn expression was positive in 159 of $380(41.8 \%)$ primary BC tissues and 14 of $68(20.6 \%)$ metastatic lesions. Thus, sTn had a higher expression rate in primary lesions than in metastatic lesions $(\mathrm{P}=0.001)$. $\mathrm{PD}-\mathrm{L} 1$ expression was positive in 78 of $380(20.5 \%)$ of primary BC tissues and 6 of 68 (8.8\%) metastatic lesions ( $\mathrm{P}=0.023)$ (Table 4). Representative slides of positive sTn and PD-L1 staining in metastatic BC lesions, including lymph node, pleura, lung, bone, brain, and bone marrow metastases, are shown in Figure 3. A total of 14 patients were evaluated with paired primary and metastatic lesions. Nine samples had no staining in both the primary and metastatic lesions, while 3 samples had positive sTn expression in the primary site but negative sTn expression in the metastatic site. None of our patients showed negative staining in the primary lesion but positive staining in the corresponding metastatic lesion. For PD-L1, 11 patients had negative expression in both paired primary and metastatic sites. Two samples showed positive staining in a primary site and negative staining in a metastatic site. No patients showed negative staining in a primary site and positive staining in a metastatic site (Table 5). Representative slides of positive sTn and PD-L1 staining in paired BC lesions are shown in Figure 4.

\section{Discussion}

During the research boom of cancer marker in the early 1980s, a carbohydrate antigen called sialyl-Tn was discovered, with positive expression rates ranging from $0 \%$ to $70 \%$. The sTn antigen had been discovered in various cancers, and was probably related to poor prognosis (23). Some experts believe that the inactivation or lack of Cosmc makes O-linked glycoproteins carry this truncated sTn glycans (24). As our recent study showed, Cosmc promoter hypermethylation could decrease the levels of Cosmc and increase Tn and sTn antigens expression in BC cells (21). Few studies have evaluated the role of sTn in the prognosis of patients with BC (25). Here, we assessed the expression of sTn in both primary and metastatic BC lesions. We further analyzed the prognostic impact of sTn expression and its relationship with PD-L1 expression. 
Table 1 The association of sTn expression level with clinicopathological characteristics in 380 BC patients

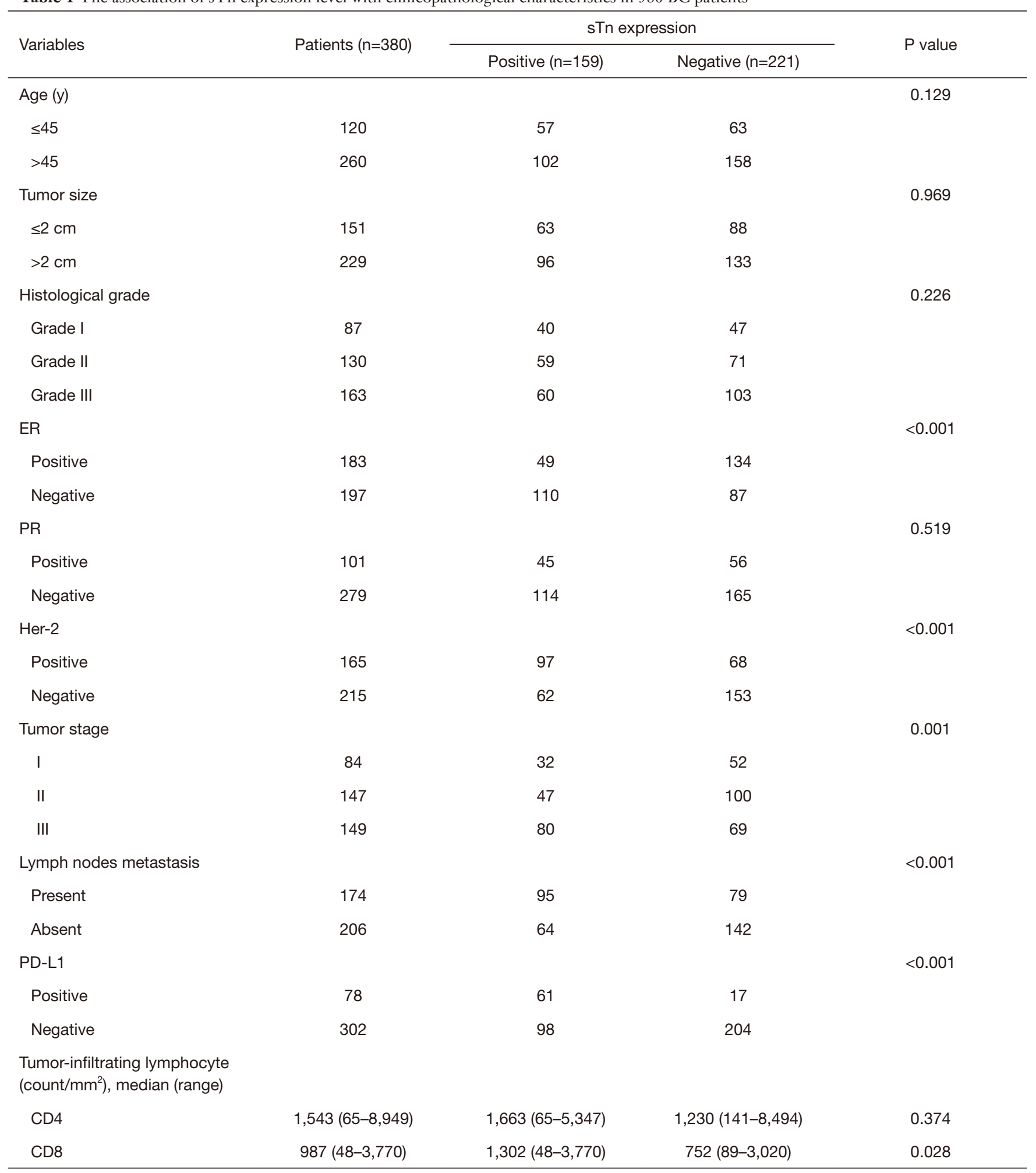



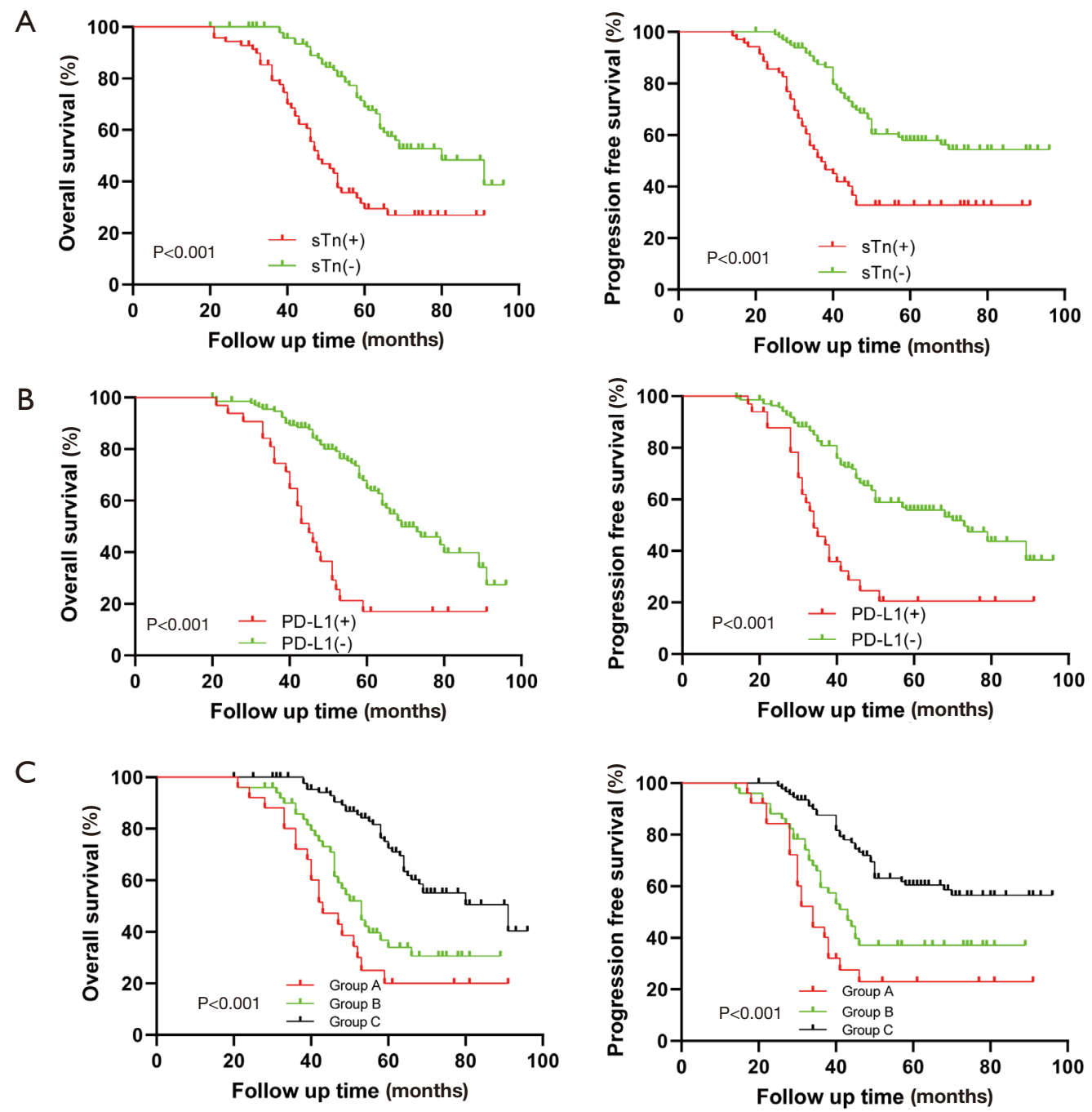

Figure 2 Kaplan-Meier survival curves for OS and PFS of patients with breast cancer according to sTn and PD-L1 expression. A, OS and PFS according to sTn expression in the training cohort and validation cohort. B, OS and PFS according to PD-L1 expression in the training cohort and validation cohort. C, OS and PFS according to a combination of sTn/PD-L1 co-expression. Group A: sTn(+)/PD-L1(+); Group B: sTn(+)/PD-L1(-) or s'Tn(-)/PD-L1(+); Group C: sTn(-)/PD-L1(-). OS, overall survival; PFS, progression-free survival.

According to our cohort of 380 patients, we assessed sTn expression patterns and analyzed the clinical significance of $\mathrm{s}$ Tn in BC. Our outcome showed that the expression rate of sTn in BC was over $40 \%$, which was related to negative ER status, positive HER-2 status, lymph node metastasis, and aggressive tumor stage. Moreover, high sTn expression was related to shorter PFS and OS of BC, corresponding with the previous work (26). Furthermore, high sTn expression was an independent prognostic factor when ER/PR/HER2 status has been adjusted. Notably, sTn and PD-L1 expression in tumor tissues demonstrated a high correlation in both cohorts of BC patients.

For BC, sTn is more highly expressed than PD-L1 (41.8\% vs. $20.5 \%)$. Both sTn and PD-L1 are more highly expressed in primary lesions than in metastatic lesions in BC samples. Our study found PD-L1 expression in 78 of 380 $(20.5 \%)$ primary sites and 6 of $68(8.8 \%)$ metastatic sites, which corresponded with prior work. Ashley and colleagues confirmed the expression rate of PD-L1 in 9 of 43 (21\%) primary tumors and 2 of $26(8 \%)$ metastatic tumors (22). In another study, there was an almost total absence of PDL1 expression in distant metastases than BC and lymph 
Table 2 Univariate analyses of prognostic factors correlated with OS and PFS

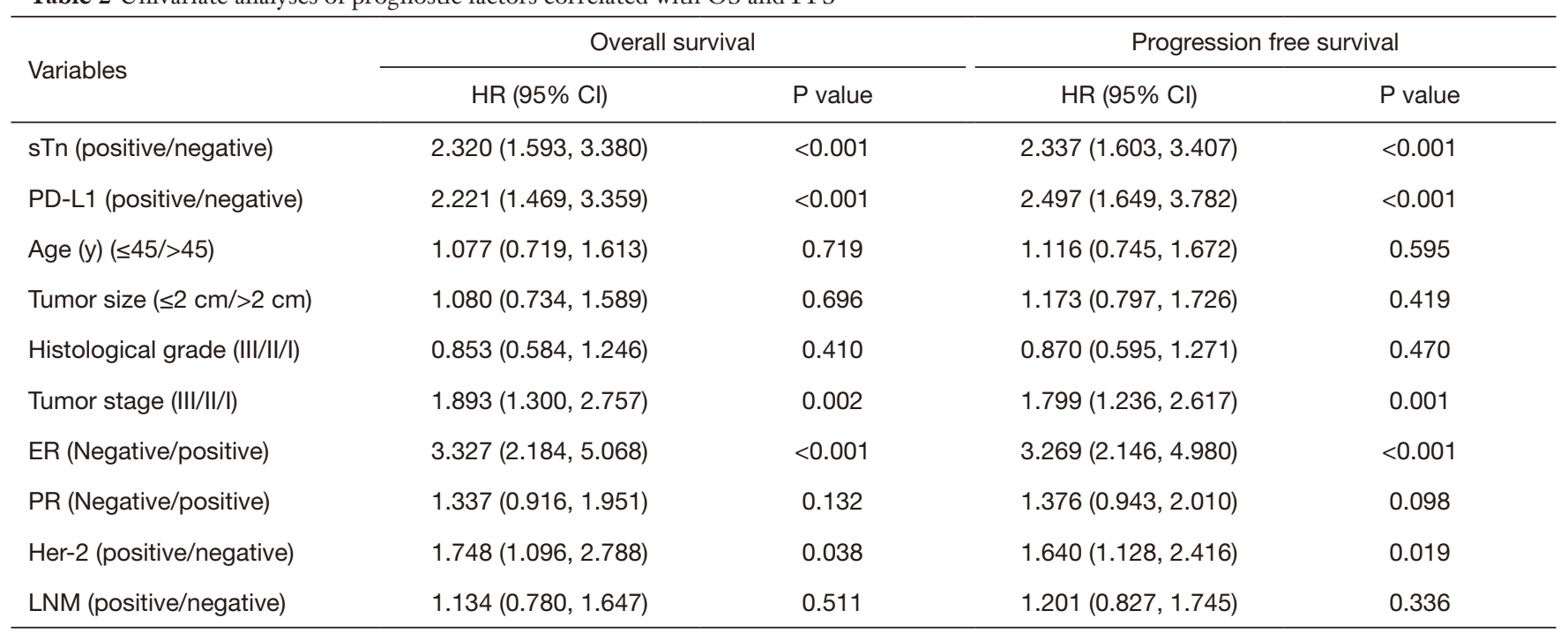

Table 3 Multivariate analyses of prognostic factors correlated with OS and PFS

\begin{tabular}{|c|c|c|c|c|}
\hline Variables & \multicolumn{2}{|c|}{ Overall survival } & \multicolumn{2}{|c|}{ Progression free survival } \\
\hline sTn (positive/negative) & $1.941(1.168,3.223)$ & 0.028 & $1.739(1.063,2.847)$ & 0.010 \\
\hline PD-L1 (positive/negative) & $1.912(1.138,3.212)$ & 0.017 & $1.863(1.116,3.110)$ & 0.014 \\
\hline ER (Negative/positive) & $0.699(0.241,2.023)$ & 0.509 & $0.662(0.232,1.888)$ & 0.440 \\
\hline Tumor stage (III/II/I) & $1.805(1.062,3.065)$ & 0.029 & $1.940(1.133,3.322)$ & 0.016 \\
\hline
\end{tabular}

Table 4 Expression of sTn and PD-L1 in metastatic breast cancer from various organ sites in the validation cohort

\begin{tabular}{|c|c|c|c|c|c|c|}
\hline & \multicolumn{3}{|c|}{ sTn expression } & \multicolumn{3}{|c|}{ PD-L1 expression } \\
\hline Lymph node & 5 & 20 & 25 & 4 & 21 & 25 \\
\hline Pleura & 3 & 4 & 7 & 1 & 6 & 7 \\
\hline Lung & 3 & 11 & 14 & 1 & 13 & 14 \\
\hline Brain & 1 & 3 & 4 & 0 & 4 & 4 \\
\hline Bone & 1 & 8 & 9 & 0 & 9 & 9 \\
\hline Marrow & 1 & 2 & 3 & 0 & 3 & 3 \\
\hline Skin & 0 & 3 & 3 & 0 & 3 & 3 \\
\hline
\end{tabular}



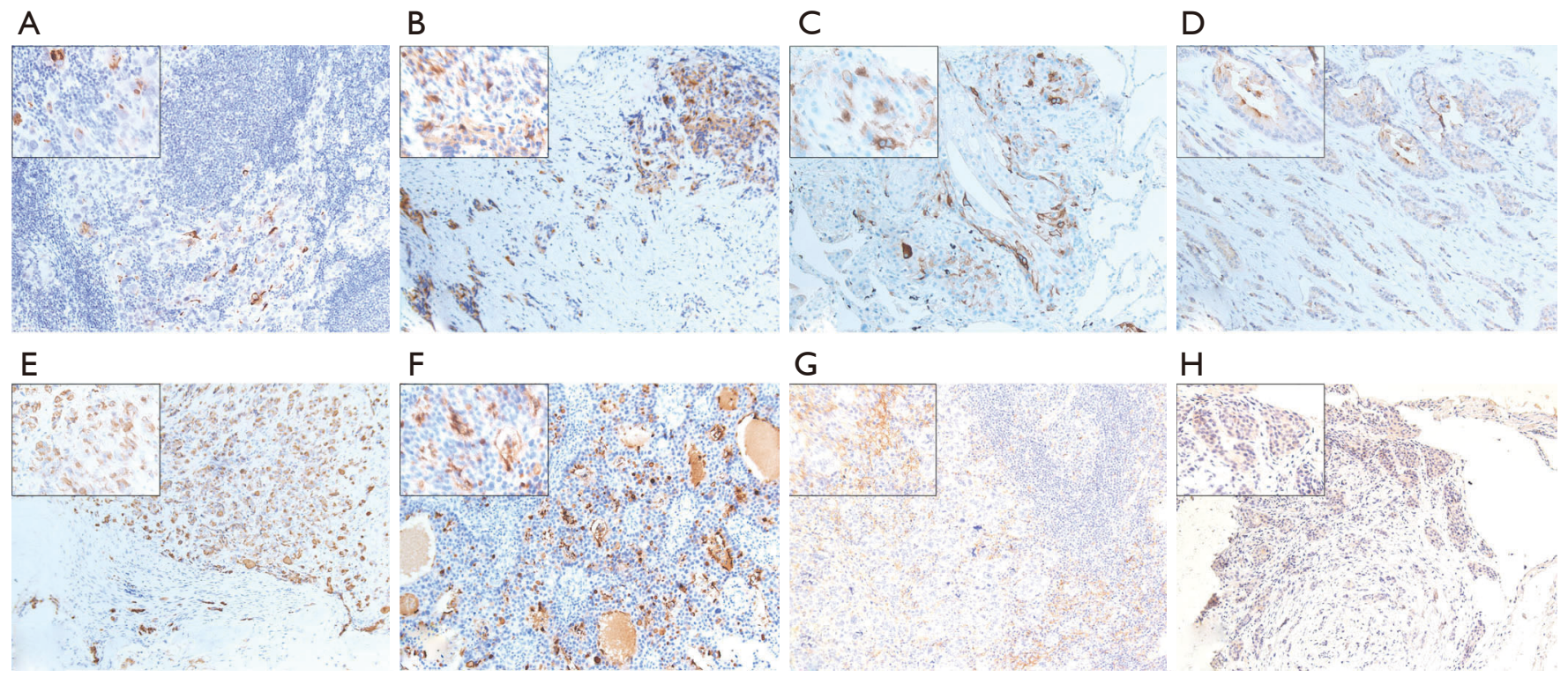

Figure 3 Representative examples of sTn and PD-L1 expression in metastatic breast cancer. (A) sTn expression in lymph node metastases. (B) sTn expression in pleura metastases. (C) sTn expression in lung metastases. (D) sTn expression in femur metastases. (E) sTn expression in bone marrow metastases. (F) s'Tn expression in brain metastases. (G) PD-L1 expression in lymph node metastases. (H) PD-L1 expression in pleura metastases. Insets show staining at $\times 40$ magnification.

Table 5 STn and PD-L1 expression in BC patients with paired primary and metastatic lesions

\begin{tabular}{lccc}
\hline Primary & Metastatic & sTn & PD-L1 \\
\hline Positive & Positive & 2 & 1 \\
Positive & Negative & 3 & 2 \\
Negative & Positive & 0 & 0 \\
Negative & Negative & 9 & 11 \\
Total patients & & 14 & 14 \\
\hline
\end{tabular}

nodes (27). Dill et al. found that positive PD-L1 expression was present in $12.4 \%(30 / 241)$ of primary BC lesions and $10 \%(4 / 40)$ of metastatic tumors, determined by IHC (28). For the paired primary and metastatic lesions, sTn and PD-L1 expression were mostly negative $(9 / 14,11 / 14)$. We found that only 3 of 14 (21.4\%) patients had an inconsistent expression of sTn, and 2 of 14 (14.3\%) had inconsistent PD-L1 expression among their metastatic lesions. These data support sTn and PD-L1 targeted immunotherapy in early-stage patients rather than metastatic patients. The absence of pre-evaluation for sTn expression may have caused the sTn-KLH vaccine (Theratope) phase III clinical trial to fail in metastatic BC patients (29).

As our outcome shows, the percentage of $\sin (+) /$
PD-L1(+) was $15.3 \%(58 / 380)$, and $>30 \%$ of PD-L1(-) samples had higher expression of sTn. Given the diverse distributions between sTn and PD-L1 expression, we decided to explore the clinical feature of sTn and PD-L1 co-expression in BC. Our results showed that sTn/PDL1 co-expression was significantly related to negative ER expression, positive HER-2 status and positive lymph node metastasis, and could be used as an independent prognostic factor for shorter PFS and OS. Our outcome provided some practical guidance for immunotherapy, as patients who test positive for sTn and PD-L1 may receive treatment related to the dual blockade of sTn and PD-L1. To confirm our assumption, we need to design more relevant clinical trials for $\mathrm{BC}$ patients. 
PBC1
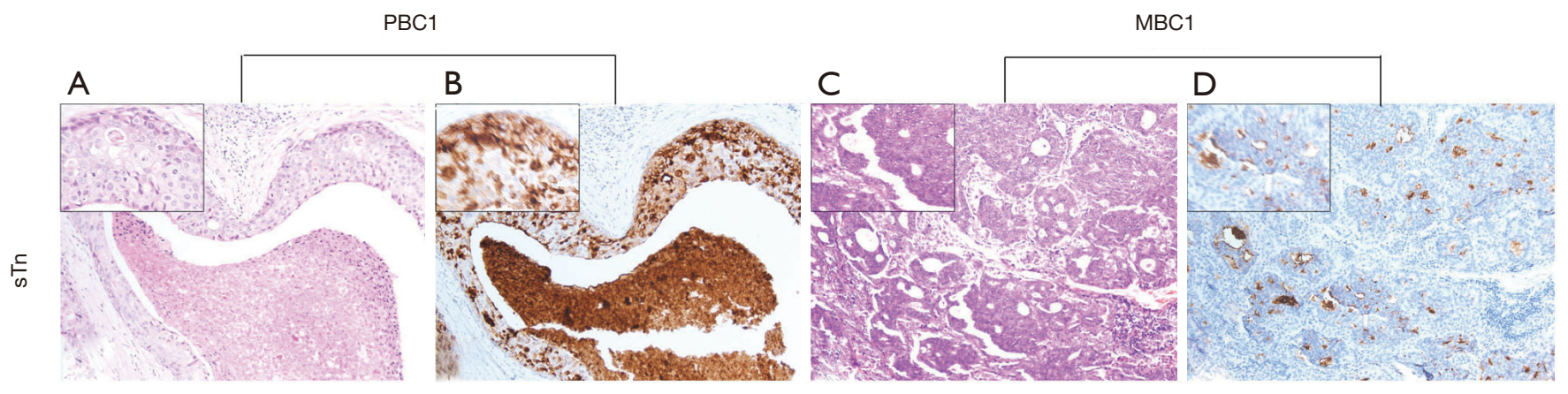

PBC2
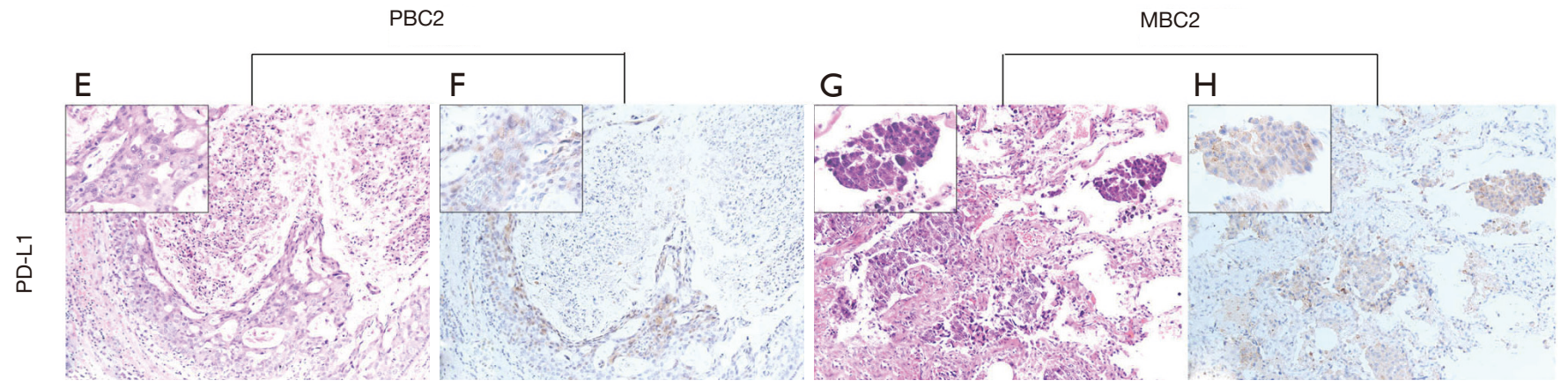

Figure 4 STn and PD-L1 expression in two representative matched PBC-MBC. (A, C, E and G) are H\&E-stained images. (B and D) show sTn expression. ( $\mathrm{F}$ and $\mathrm{H}$ ) show PD-L1 expression. PBC1 and MBC1 are from the same patient. PBC2 and MBC2 are from the same patient. MBC1 and MBC2 are lung metastases. Insets show staining at $\times 40$ magnification. PBC, primary breast cancer; MBC, metastatic breast cancer.

In recent years, there have been many related studies presenting the immunosuppressive effect of the sTn antigen, which binds to receptors on various immune cells, such as dendritic cells (DCs), natural killer (NK) cells, and macrophages. For DC maturation and apoptosis, Carrascal et al. determined that sTn antigen expression in bladder cancer cells could suppress DC maturation and impede T cell activation (30). Another study (31) reported that the binding of soluble Siglec- 3 with the sTn antigen could partially induce DC apoptosis. Furthermore, Takamiya et al. indicated that Siglec-15 could identify the sTn antigen in tumor cells, and transduce a signal to enhance TGF- $\beta$ secretion in tumor-associated macrophages (32). In addition, Ogata et al. demonstrated that mucins linked with the tumoral sTn antigen could effectively suppress NK cell cytotoxicity (33). According to our outcome, the overexpression of sTn was significantly related to PD-L1 overexpression and a high density of $\mathrm{CD} 8^{+}$TILs, rather than $\mathrm{CD}^{+}$TILs in BC. These data suggested that cancer cells with sTn expression tended to trigger immune evasion and tolerance (34).

However, our research still involved some limitations.
Firstly, our study was a retrospective study and a prospective multicenter study should be carried out in the future. Secondly, we only explored the relationship between sTn and PD-L1 in a clinical study. This work should be further expanded to cell experiments to study the molecular mechanisms of both markers. Finally, we only used tumor cells as the research objects when we evaluated PD-L1 expression. Considering that the expression of PD-L1 in immune cells has clinical importance and has become more prevalent immune cells should also be used as research objects and analyzed accordingly.

In summary, our work first put forward the positive relevance between sTn expression and PD-L1 expression in BC tissues. The co-expression of sTn/PD-L1 was an independent predictive factor of poor prognosis. Therefore, combinational therapy with dual blockade might benefit BC patients with $\mathrm{s} \operatorname{Tn}(+) / \mathrm{PD}-\mathrm{L} 1(+)$ expression, which requires testing in future clinical trials.

\section{Acknowledgments}

Funding: This study was supported by the Youth Project 
of National Natural Science Foundation of China (NO. 81702587) and the project of Beijing Municipal Science and Technology Commission (NO. Z181100002218001).

\section{Footnote}

Reporting Checklist: The authors have completed the REMARK reporting checklist. Available at https://dx.doi. org/10.21037/gs-21-83

Data Sharing Statement: Available at https://dx.doi. org/10.21037/gs-21-83

Conflicts of Interest: All authors have completed the ICMJE uniform disclosure form (available at https://dx.doi. org/10.21037/gs-21-83). The authors have no conflicts of interest to declare.

Ethical Statement: The authors are accountable for all aspects of the work in ensuring that questions related to the accuracy or integrity of any part of the work are appropriately investigated and resolved. The study was conducted in accordance with the Declaration of Helsinki (as revised in 2013). The study was approved by institutional ethics board of Beijing Chaoyang Hospital (NO.: 2020-316-13) and the participants gave informed consent before taking part.

Open Access Statement: This is an Open Access article distributed in accordance with the Creative Commons Attribution-NonCommercial-NoDerivs 4.0 International License (CC BY-NC-ND 4.0), which permits the noncommercial replication and distribution of the article with the strict proviso that no changes or edits are made and the original work is properly cited (including links to both the formal publication through the relevant DOI and the license). See: https://creativecommons.org/licenses/by-nc-nd/4.0/.

\section{References}

1. Siegel RL, Miller KD, Jemal A. Cancer statistics, 2019. CA Cancer J Clin 2019;69:7-34.

2. Emens LA. Breast Cancer Immunotherapy: Facts and Hopes. Clin Cancer Res 2018;24:511-20.

3. Kammerer-Jacquet SF, Deleuze A, Saout J, et al. Targeting the PD-1/PD-L1 Pathway in Renal Cell Carcinoma. Int J Mol Sci 2019;20:1692.

4. Bastaki S, Irandoust M, Ahmadi A, et al. PD-L1/PD-1 axis as a potent therapeutic target in breast cancer. Life Sci 2020;247:117437.

5. Koomen BM, Badrising SK, van den Heuvel MM, et al. Comparability of PD-L1 immunohistochemistry assays for non-small-cell lung cancer: a systematic review. Histopathology 2020;76:793-802.

6. Bence C, Hofman V, Chamorey E, et al. Association of combined PD-L1 expression and tumour-infiltrating lymphocyte features with survival and treatment outcomes in patients with metastatic melanoma. J Eur Acad Dermatol Venereol 2020;34:984-94.

7. Herbst RS, Soria JC, Kowanetz M, et al. Predictive correlates of response to the anti-PD-L1 antibody MPDL3280A in cancer patients. Nature 2014;515:563-7.

8. Planes-Laine G, Rochigneux P, Bertucci F, et al. PD-1/ PD-L1 Targeting in Breast Cancer: The First Clinical Evidences Are Emerging. A Literature Review. Cancers 2019;11:1033.

9. Munkley J. The Role of Sialyl-Tn in Cancer. Int J Mol Sci 2016;17:275.

10. Beatson R, Maurstad G, Picco G, et al. The Breast CancerAssociated Glycoforms of MUC1, MUC1-Tn and sialylTn, Are Expressed in COSMC Wild-Type Cells and Bind the C-Type Lectin MGL. PloS One 2015;10:e0125994.

11. Conze T, Carvalho AS, Landegren U, et al. MUC2 mucin is a major carrier of the cancer-associated sialyl-Tn antigen in intestinal metaplasia and gastric carcinomas. Glycobiology 2010;20:199-206.

12. Ikeda Y, Kuwano H, Baba K, et al. Expression of Sialyl-Tn antigens in normal squamous epithelium, dysplasia, and squamous cell carcinoma in the esophagus. Cancer Res 1993;53:1706-8.

13. Terasawa K, Furumoto H, Kamada M, et al. Expression of Tn and sialyl-Tn antigens in the neoplastic transformation of uterine cervical epithelial cells. Cancer Res 1996;56:2229-32.

14. Hakomori S. Tumor-associated carbohydrate antigens defining tumor malignancy: basis for development of anticancer vaccines. Adv Exp Med Biol 2001;491:369-402.

15. Xu F, Fan C, Fan S, et al. Expression profile of mucinassociated sialyl-Tn antigen in Chinese patients with different colorectal lesions (adenomas, carcinomas). Int J Clin Exp Pathol 2015;8:11549-54.

16. Eavarone DA, Al-Alem L, Lugovskoy A, et al. Humanized anti-Sialyl-Tn antibodies for the treatment of ovarian carcinoma. PloS One 2018;13:e0201314.

17. Thomas D, Sagar S, Caffrey T, et al. Truncated O-glycans promote epithelial-to-mesenchymal transition and 
stemness properties of pancreatic cancer cells. J Cell Mol Med 2019;23:6885-96.

18. Boraston AB, Bolam DN, Gilbert HJ, et al. Carbohydratebinding modules: fine-tuning polysaccharide recognition. Biochem J 2004;382:769-81.

19. Beatson R, Tajadura-Ortega V, Achkova D, et al. The mucin MUC1 modulates the tumor immunological microenvironment through engagement of the lectin Siglec-9. Nat Immunol 2016;17:1273-81.

20. Rodriguez E, Boelaars K, Brown K, et al. Sialic acids in pancreatic cancer cells drive tumour-associated macrophage differentiation via the Siglec receptors Siglec-7 and Siglec-9. Nat Commun 2021;12:1270.

21. Xu F, Wang D, Cui J, et al. Demethylation of the Cosmc Promoter Alleviates the Progression of Breast Cancer Through Downregulation of the Tn and Sialyl-Tn Antigens. Cancer Manag Res 2020;12:1017-27.

22. Cimino-Mathews A, Thompson E, Taube JM, et al. PD-L1 (B7-H1) expression and the immune tumor microenvironment in primary and metastatic breast carcinomas. Hum Pathol 2016;47:52-63.

23. Julien S, Videira PA, Delannoy P. Sialyl-tn in cancer: (how) did we miss the target? Biomolecules 2012;2:435-66.

24. Ju T, Lanneau GS, Gautam T, et al. Human tumor antigens $\mathrm{Tn}$ and sialyl $\mathrm{Tn}$ arise from mutations in Cosmc. Cancer Res 2008;68:1636-46.

25. Kinney AY, Sahin A, Vernon SW, et al. The prognostic significance of sialyl-Tn antigen in women treated with breast carcinoma treated with adjuvant chemotherapy. Cancer 1997;80:2240-9.

26. Leivonen M, Nordling S, Lundin J, et al. STn and prognosis in breast cancer. Oncology 2001;61:299-305.

Cite this article as: $\mathrm{Xu} \mathrm{F,} \mathrm{Zhao} \mathrm{H,} \mathrm{Li} \mathrm{J,} \mathrm{Jiang} \mathrm{H.} \mathrm{Mucin-type}$ sialyl-Tn antigen is associated with PD-L1 expression and predicts poor clinical prognosis in breast cancer. Gland Surg 2021;10(7):2159-2169. doi: 10.21037/gs-21-83
27. Tawfik O, Kimler BF, Karnik T, et al. Clinicopathological correlation of PD-L1 expression in primary and metastatic breast cancer and infiltrating immune cells. Hum Pathol 2018;80:170-8.

28. Dill EA, Dillon PM, Bullock TN, et al. IDO expression in breast cancer: an assessment of 281 primary and metastatic cases with comparison to PD-L1. Mod Pathol 2018;31:1513-22.

29. Miles D, Roché H, Martin M, et al. Phase III multicenter clinical trial of the sialyl-TN (STn)-keyhole limpet hemocyanin $(\mathrm{KLH})$ vaccine for metastatic breast cancer. Oncologist 2011;16:1092-100.

30. Carrascal MA, Severino PF, Guadalupe Cabral M, et al. Sialyl Tn-expressing bladder cancer cells induce a tolerogenic phenotype in innate and adaptive immune cells. Mol Oncol 2014;8:753-65.

31. Ishida A, Ohta M, Toda M, et al. Mucin-induced apoptosis of monocyte-derived dendritic cells during maturation. Proteomics 2008;8:3342-9.

32. Takamiya R, Ohtsubo K, Takamatsu S, et al. The interaction between Siglec-15 and tumor-associated sialylTn antigen enhances TGF- $\beta$ secretion from monocytes/ macrophages through the DAP12-Syk pathway. Glycobiology 2013;23:178-87.

33. Ogata S, Maimonis PJ, Itzkowitz SH. Mucins bearing the cancer-associated sialosyl-Tn antigen mediate inhibition of natural killer cell cytotoxicity. Cancer Res 1992;52:4741-6.

34. Ohno S, Ohno Y, Nakada H, et al. Expression of Tn and sialyl-Tn antigens in endometrial cancer: its relationship with tumor-produced cyclooxygenase-2, tumor-infiltrated lymphocytes and patient prognosis. Anticancer Res 2006;26:4047-53. 


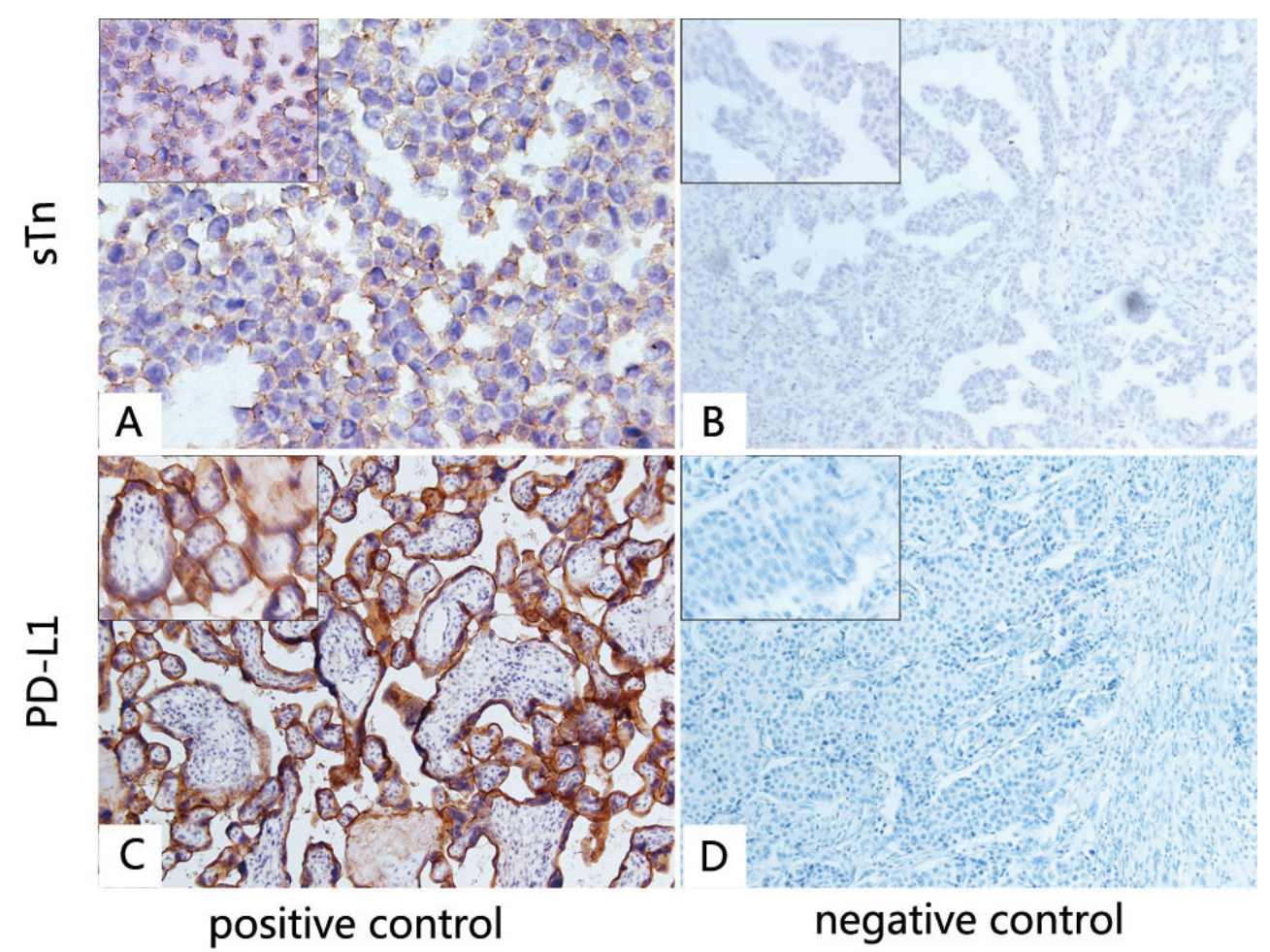

Figure S1 Positive controls and negative controls for sTn and PD-L1 staining. (A) sTn expression in Cosmc-KO MCF-7 breast cancer cells. (C) PD-L1 expression in human placenta tissues. (B and D) were prepared by replacing the primary antibody with PBS. Insets show staining at $\times 40$ magnification. 